\title{
Achievable hierarchies in voting games with abstention
}

\author{
Josep Freixas *, Bertrand Tchantcho ${ }^{\dagger}$ and Narcisse Tedjeugang ${ }^{\ddagger}$
}

November 19, 2013

\begin{abstract}
It is well known that he influence relation orders the voters the same way as the classical Banzhaf and Shapley-Shubik indices do when they are extended to the voting games with abstention (VGA) in the class of complete games. Moreover, all hierarchies for the influence relation are achievable in the class of complete VGA. The aim of this paper is twofold. Firstly, we show that all hierarchies are achievable in a subclass of weighted VGA, the class of weighted games for which a single weight is assigned to voters. Secondly, we conduct a partial study of achievable hierarchies within the subclass of H-complete games, that is, complete games under stronger versions of influence relation.
\end{abstract}

Keywords : Game theory · $(3,2)$ Voting rules · Abstention · Decision Support Systems · Weightedness and Completeness $\cdot$ Hierarchies

Mathematics Subject Classification (2000) 91A12 · 05C65 - 94C10

\footnotetext{
* "Departament de Matemàtica Aplicada 3 i Escola Politècnica Superior d'Enginyeria de Manresa, Universitat Politècnica de Catalunya". Research partially supported by "Ministerio de Economía y Competitividad proyecto MTM2012-34426/FEDER" and "Govern de la Generalitat SGR 2009-1029" ; josep.freixas@upc.edu.

${ }^{\dagger}$ Corresponding author : btchantcho@yahoo.fr, University of Yaounde I ; MASS laboratory - University of Cergy Pontoise ; THEMA laboratory.

†tedjeugang@yahoo.fr, University of Yaounde I.
} 


\section{Introduction}

In a committee, a voting rule specifies the decision-making procedure, that is, when a proposal is to be accepted or rejected depending on the resulting vote configuration. The vote configuration itself depends on different options offered to committee members. A very huge class of voting rules studied in the literature deals with simple games. In such voting models, any voter either votes for or against the proposal. If a voter does not favor a proposal, then he is considered to be against, that is, an abstention if any, is treated as a vote against the proposal. It is well known however that many decision-making processes including relative majority, vote in the United Nations Senate cannot be fitted in such models.

To handle this shortcoming, models of voting games with abstention (VGA) were introduced (Rubinstein [26] defined social decision systems, Felsenthal and Machover ([9] and [10]) defined Ternary Voting Game), where each player is allowed three distinct votes but the outcome of the vote still has two options. An important but isolated earlier work on abstention can be found in Fishburn's book [11]. More recently, Freixas and Zwicker [12] extended VGAs to the so-called $(j, k)$ games, a subclass of which are $(j, 2)$ games in which voters have $j$ possible ordered levels of approval in the input, thus partitioning the committee $N$ into $j$ coalitions, each attached to a winning or losing character in the output. Simple games constitute the class of $(2,2)$ simple games whereas VGAs constitute the class of $(3,2)$ simple games.

A fundamental question is the assessment of the influence of each voter to affect the outcome of a vote. Several power indices for simple games have so far been defined to capture the ability of the players to affect the voting outcome. The two most conspicuous representatives of this line of research are the Shapley-Shubik (SS) power index [27] and the Banzhaf and Coleman (BC) power indices ([2] and [6]) originally defined in voting rules modelled by simple games. In a quite distinct direction, the desirability relation (introduced by Isbell [21] and extensively studied by Taylor [29]) rank directly players according to their influence. Previous work by Felsenthal and Machover [9] and Diffo Lambo and Moulen [8] show that all these power theories are ordinally equivalent in the class of swap-robust simple games.

In order to capture the ordering of the influence held by the players in a game, the concept of hierarchy was introduced by Friedman et al [20]. For example, a five-player game $\mathrm{G}$ has hierarchy $(1,3,1)$ means that one player has less influence than all the others, one player has more, and the other three players have the same influence as each other, they are equivalent. From works by Friedman et al [20] and Freixas and Pons [17] on hierarchies, it can be stated that given any 
complete pre-ordering defined on a finite set of more than 5 voters, it is possible to construct a simple game such that the pre-orderings induced by SS [27] and the BColeman ([2] and [6]) power indices coincide with the given pre-ordering (when the number of voters is 5 or less, there are four non achievable hierarchies). These results hold under the condition that the game be modeled by a simple game. With respect to the construction of the hierarchies, recently, Bishnu and Roy [4] have shown how to use minimal winning coalitions to extract the hierarchy of players.

This paper deals with voting games with abstention. The question of achievable hierarchies is relevant thanks to the fact that SS and BC indices have all been clearly generalized to VGA by Felsenthal and Machover [9] while the Coleman index has been generalized to VGA by Freixas [15]. They have moreover been generalized to the most general model of $(j, k)$ games by Freixas ([13] and [14]). On the other hand, the desirability relation has been defined by Tchantcho et al [31] in terms of $I$-influence relation. These authors showed that the SS, BC and the $I$-influence relation are ordinally equivalent in the subclass of equitable swap-robust games. Recently, Parker [24] showed that this ordinal equivalence holds in the whole class of swap-robust games.

With respect to hierarchies, we show in this paper that all hierarchies are achievable. More precisely, the four hierarchies cited above that were not achievable for simple games (when abstentions are not permitted to players) are achievable in a particular class of weighted games, the class of zero-centered strongly weighted VGA. This is a refinement of Parker's result [24]. Weighted games as well as the characterization of this class of games were given by Freixas and Zwicker in [12].

Freixas et al [18] noticed some shortcoming in the $I$-influence. There are weighted games not being complete for the influence relation, something different to what occurs for simple games. They introduced several extensions of the desirability relation (see also Pongou et al [25]) by considering each condition in the definition of $I$-influence relation. A stronger form of $I$-completeness is $H$-completeness for which all the relations that intervene in the definition of completeness coincide. In this paper we also address the problem of achievable $H$-hierarchies. We show in particular that no strict hierarchy is achievable for games with 2 or 3 players. For games with 4 players, except the strict hierarchy for which we do not get any answer, all other hierarchies are achievable. For games with more than 5 players, the strict hierarchy is achievable in the class of $H$-complete $(3,2)$ games. Furthermore, unlike the subclass of hierarchies $(m, 1,1)$ with $m \geq 2$, all other $H$-hierarchies are achievable.

Determining importance rankings is a significant issue in operational research. 
The study of ordinal preferences involves a variety of fields, including tournament theory, multiple criteria decision modeling (MCDM), and data envelopment analysis of qualitative data. As stated in the survey by Cook [7], the notion of voter power or relative importance has been largely ignored in studies on ordinal ranking problems, although if a tangible estimate of voter importance exists, then these voters can be treated like criteria in an MCDM problem. The approach in our paper is useful in ranking voters in voting institutions where abstention is allowed as a third input. Examples of application of our results naturally apply to political institutions, but also in management enterprisers and even in reliability systems where voters are replaced by device components with three input levels. Examples in these different contexts can be found in: Levitin [22], Obata and Ishii [23], Alonso-Meijide et al. [1], Sueyoshi et al. [28] or Freixas et al. [16]. The paper is organized as follows.

The technical background as well as some useful results are recalled in section 2. In section 3 , we recall several notion of desirability for $(3,2)$ games and consider as well their completeness, their link with weightedness. In section 4 we prove that all hierarchies induced by the influence relation are achievable in the particular class of weighted $(3,2)$ games, the class of zero-centered strongly weighted VGA. As for $H$-hierarchies a partial study is done in section 5 and a conclusion then ends the paper.

\section{Preliminaries}

An ordered 3-partition of $N$ (set of voters or players) is a sequence $S=\left(S_{1}, S_{2}, S_{3}\right)$ of mutually disjoint subsets of $N$ whose union is $N$. In $S, S_{1}$ stands for the set of yes voters, $S_{2}$ for abstainers and $S_{3}$ stands for no voters. We denote by $3^{N}$ the set of all ordered 3-partitions of $N$. For $S, S^{\prime} \in 3^{N}$, we write $S \subset^{3} S^{\prime}$ if $S$ can be transformed into $S^{\prime}$ by shifting one or more voters to higher levels of approval.

Definition 2.1 $A(3,2)$ game $G=(N, V)$ consists of a finite set $N$ of voters together with a value function $V: 3^{N} \longrightarrow\{0,1\}$ such that for all ordered 3-partition $S, S^{\prime}$, if $S \subset^{3} S^{\prime}$ then $V(S)=1$ implies $V\left(S^{\prime}\right)=1$.

A 3-partition $S$ such that $V(S)=1$ is said to be winning. A $(3,2)$ game can be defined by its set of winning 3-partitions, $W=\left\{S \in 3^{N}: V(S)=1\right\}$. In that case we denote the game by $(N, W)$. In voting, it is often demanded that $V$ be exhaustive, then from the monotonicity demanded to $V, V(\mathcal{N})=0$ and $V(\mathcal{M})=1$ where $\mathcal{N}$ and $\mathcal{M}$ are respectively the 3-partitions with $\mathcal{N}_{3}=N$ and $\mathcal{M}_{1}=N$. A special type of $(3,2)$ simple games is the class of anonymous or symmetric games which have been intensively studied in [19]. Anonymous (3,2) 
games are games for which for all 3-partition $S, S$ is winning if and only if for all permutations $\pi: N \rightarrow N, \pi(S)=\left(\pi\left(S_{1}\right), \pi\left(S_{2}\right), \pi\left(S_{3}\right)\right)$ is winning.

Definition 2.2 In a $(3,2)$ game, a 3-partition $S$ is said minimal winning if $S$ is winning and for all $T \in 3^{N}$ such that $T \subset^{3} S, T$ is losing. As well, $S$ is said maximal losing if $S$ is losing and for all $T \in 3^{N}$ such that $S \subset^{3} T, T$ is winning.

Either the set of minimal winning 3-partitions or the set of maximal losing 3 -partitions completely generates the game. Next, we introduce weighted $(3,2)$ games, which is a special type of weighted $(j, k)$ games introduced in [12].

Definition 2.3 Let $G=(N, V)$ be a $(3,2)$ game. A representation of $G$ as a $(3,2)$ weighted game consists of a sequence $w=\left(w_{1}, w_{2}, w_{3}\right)$ of 3 weight functions, where $w_{i}: N \longrightarrow \mathbb{R}$ for each $i$ with $w_{1}(p) \geq w_{2}(p) \geq w_{3}(p)$ for each $p \in N$, together with a real number $Q$ so called quota such that for any 3-partition $S, S$ is winning if and only if $w(S)=\sum_{i=1}^{3} \sum_{p \in S_{i}} w_{i}(p) \geq Q$.

According to this definition we can normalize, i.e. assign a zero weight, to any level of approval. Here we are mainly concerned with games with abstention for which we can normalize the weights at any of the three input levels, but we choose the "abstention" level which seems to be quite natural. If a null weight is assigned to abstainers, then a non-negative weight is assigned to "yes" voters and a non-positive weight to "no" voters. Thus, a weight ${ }^{1} w(p)=\left(w^{+}(p), 0, w^{-}(p)\right)$ with $w^{+}(p) \geq 0$ and $w^{-}(p) \leq 0$ is assigned to each $p \in N$. The only requirement for the threshold $Q$, if the $(3,2)$ game is demanded to be exhaustive, is

$$
w(\mathcal{N})=w(\emptyset, \emptyset, N)=\sum_{p \in N} w^{-}(p)<Q \leq \sum_{p \in N} w^{+}(p)=w(N, \emptyset, \emptyset)=w(\mathcal{M})
$$

The previous definition can now be rewritten as follows.

Definition $2.4 A(3,2)$ game $(N, W)$ is a weighted $(3,2)$ game if there exists a sequence of weight functions $\left(w^{+}, 0, w^{-}\right)$with $w^{-}(p) \leq 0 \leq w^{+}(p)$ for all $p \in N$, and a quota $Q$ such that for all $S=\left(S_{1}, S_{2}, S_{3}\right) \in 3^{N}$,

$$
S \in W \Longleftrightarrow w(S)=\sum_{p \in S_{1}} w^{+}(p)+\sum_{p \in S_{3}} w^{-}(p) \geq Q
$$

Two consecutive stronger conditions of a weighted $(3,2)$ game are the two following which were introduced in [12] :

\footnotetext{
${ }^{1}$ We are identifying $w^{+}$with $w_{1}, 0$ with $w_{2}$ and $w^{-}$with $w_{3}$ in Definition 2.4.
} 
Definition 2.5 - A strongly weighted $(3,2)$ game is a weighted $(3,2)$ game that admits a representation such that for every pair of voters $p$ and $r$,

$\left[w^{+}(p) \geq w^{+}(r),-w^{-}(p) \geq-w^{-}(r)\right]$ or $\left[w^{+}(p) \leq w^{+}(r),-w^{-}(p) \leq-w^{-}(r)\right]$.

- A zero-centered strongly weighted $(3,2)$ game is a strongly weighted $(3,2)$ game that admits a representation with weights $w^{+}(p)=-w^{-}(p)$ for each $p \in N$.

\section{Various influence relations on the set of voters}

We recall here the influence relation defined in [31].

Definition 3.1 Let $G=(N, V)$ be a $(3,2)$ game, $p, r \in N: p$ is said to be at least as influential as $r$, denoted $p \succsim_{I} r$, if for all $\left(S_{1}, S_{2}, S_{3}\right) \in 3^{N}$ it yields :

- $V\left(S_{1} \cup\{p\}, S_{2} \backslash\{p\}, S_{3}\right) \geq V\left(S_{1} \cup\{r\}, S_{2} \backslash\{r\}, S_{3}\right)$ if $p, r \in S_{2}$,

- $V\left(S_{1}, S_{2} \cup\{p\}, S_{3} \backslash\{p\}\right) \geq V\left(S_{1}, S_{2} \cup\{r\}, S_{3} \backslash\{r\}\right)$ if $p, r \in S_{3}$, and

- $V\left(S_{1} \cup\{p\}, S_{2}, S_{3} \backslash\{p\}\right) \geq V\left(S_{1} \cup\{r\}, S_{2}, S_{3} \backslash\{r\}\right)$ if $p, r \in S_{3}$.

$G$ is I-complete if either $p \succsim_{I} r$ or $r \succsim_{I} p$ for all pair $p, r \in N$.

The $I$-influence relation which is clearly a generalization of the desirability relation for simple games to $(3,2)$ games, is reflexive, but is neither complete nor transitive in general. However, there exist weighted $(3,2)$ games that are not complete under the $I$-influence relation. Although $I$-completeness is not consistent for the notion of weighted $(3,2)$ games, it was shown in [18] that it is for the notion of strongly weighted $(3,2)$ games. The $I$-influence is indeed too demanding. In [18], the three separate condition of the $I$-influence has been studied thus introducing a new class of complete games.

Definition 3.2 Let $(N, V)$ be a $(3,2)$ game. Let $p, r \in N$ :

(i) $D^{+}$-desirability. $p \succsim_{D^{+}} r$ if and only if for all 3-partition $S=\left(S_{1}, S_{2}, S_{3}\right)$ such that both $p$ and $r$ belong to $S_{2}, V\left(S_{1} \cup\{p\}, S_{2} \backslash\{p\}, S_{3}\right) \geq V\left(S_{1} \cup\right.$ $\left.\{r\}, S_{2} \backslash\{r\}, S_{3}\right)$.

(ii) $D^{-}$-desirability. $p \succsim_{D^{-}} r$ if and only if for all 3-partition $S=\left(S_{1}, S_{2}, S_{3}\right)$ such that both $p$ and $r$ belong to $S_{3}, V\left(S_{1}, S_{2} \cup\{p\}, S_{3} \backslash\{p\}\right) \geq V\left(S_{1}, S_{2} \cup\right.$ $\left.\{r\}, S_{3} \backslash\{r\}\right)$. 
(iii) $D^{ \pm}$-desirability. $p \succsim_{D^{ \pm}} r$ if and only if for all 3-partition $S=\left(S_{1}, S_{2}, S_{3}\right)$ such that both $p$ and $r$ belong to $S_{3}, V\left(S_{1} \cup\{p\}, S_{2}, S_{3} \backslash\{p\}\right) \geq V\left(S_{1} \cup\right.$ $\left.\{r\}, S_{2}, S_{3} \backslash\{r\}\right)$.

Of course, $V\left(S_{1} \cup\{p\}, S_{2} \backslash\{p\}, S_{3}\right) \geq V\left(S_{1} \cup\{r\}, S_{2} \backslash\{r\}, S_{3}\right)$ is equivalent to assert that $\left(S_{1} \cup\{r\}, S_{2} \backslash\{r\}, S_{3}\right) \in W$ implies that $\left(S_{1} \cup\{p\}, S_{2} \backslash\{p\}, S_{3}\right) \in W$. And analogously for the two next inequalities in Definition 3.2.

In general, none of the three separate relations, $\succsim_{D^{+}}, \succsim_{D^{-}}$and $\succsim_{D^{ \pm}}$are transitive. The notion of completeness induced for the three separate relations considered in Definition 3.2 and two additional ones are recalled below.

Definition 3.3 Let $G=(N, V)$ a (3,2) game.

(i) $G$ is $D^{+}$-complete if either $p \succsim_{D^{+}} r$ or $r \succsim_{D^{+}} p$ for all $p, r \in N$.

(ii) $G$ is $D^{-}$-complete if either $p \succsim_{D^{-}} r$ or $r \succsim_{D^{-}} p$ for all $p, r \in N$.

(iii) $G$ is $D^{ \pm}$-complete if either $p \succsim_{D^{ \pm}} r$ or $r \succsim_{D^{ \pm}} p$ for all $p, r \in N$.

(iv) $G$ is complete if it is $D^{+}$-complete, $D^{-}$-complete and $D^{ \pm}$-complete.

(v) $G$ is hierarchically complete or $H$-complete, denoted by $\succsim_{H}$, if it is complete and the total rankings induced by $\succsim_{D^{+}}, \succsim_{D^{-}}$and $\succsim_{D^{ \pm}}$coincide.

Note that even if a $(3,2)$ game is complete the total rankings given by $\succsim_{D^{+}}$, $\succsim_{D^{-}}$and $\succsim_{D^{ \pm}}$can be different. The inclusion relations among the different classes of $(3,2)$ games considered above can be summarized as follows.

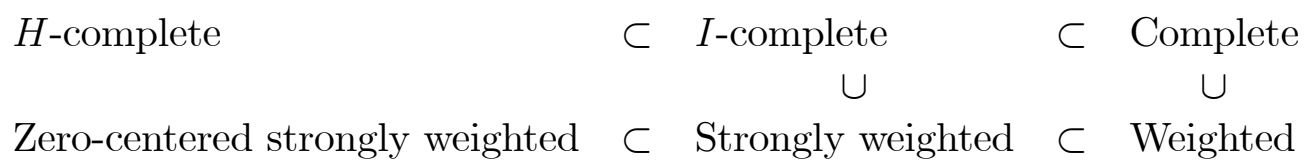

Anonymous $(3,2)$ games are the simplest subclass of $(3,2)$ games being $H$-complete and therefore $I$-complete and complete. These $(3,2)$ games are the only ones with a unique equivalence class and therefore all players are hierarchically equivalents. Somewhat curious, and contrarily to what happens for simple games, is that some $(3,2)$ anonymous games are not necessarily weighted, see [19] and [32] for a characterization of anonymous weighted $(3,2)$ games. However, if an anonymous $(3,2)$ game is weighted it is zero-centered strongly weighted.

Sections 4 and 5 are devoted to the study of achievable hierarchies for $I$-complete and $H$-complete games respectively. 


\section{$4 \quad I$-hierarchies}

This section is devoted to the study of the existence of achievable hierarchies for I-complete $(3,2)$ games. The study of hierarchies within the class of simple games can be traced back to Friedman et al. [20] and continued by Bean et al. [3], even though it is also an implicit study in Carreras and Freixas [5]. Indeed, in Friedman et al. [20] it is proved that complete simple games and, particularly, weighted simple games show many different hierarchies, although two sequences of hierarchies are never achievable. Freixas and Pons [17] proved that all hierarchies are achievable in the class of weakly complete games as long as the number of voters is greater than 5. For less than 6 voters, only four hierarchies are not achieved in this class of games, and they are : $(1,1,1),(1,1,1,1),(2,1,1)$ and $(2,1,1,1)$. But none of these four hierarchies is achieved either in any other kind of simple games. As a consequence of these results we can state that, given any complete pre-ordering defined on a finite set of voters (with more than five elements), it is possible to construct a simple game such that the pre-orderings induced by Shapley-Shubik [27] and the Banzhaf and Coleman ([2] and [6]) power indices coincide with the given pre-ordering.

Definition 4.1 Let $G=(N, W)$ be a $(3,2)$ game and $\succsim$ be a pre-ordering on $N$.

We say that the sequence $\left(m_{1}, \ldots, m_{t}\right)$ with $\sum_{i=1}^{t} m_{i}=n$ is achievable for $\succsim$ if there are $t$ equivalence classes $A_{1}, \ldots, A_{t}$ for $\succsim$ which form a partition of $N$ with $m_{1}$, ..., $m_{t}$ voters respectively and $p \succ r$ whenever $p \in A_{i}, r \in A_{j}$ with $i<j$.

Note that at most there are $2^{n-1}$ different hierarchies for games with $n$ voters. Parker [24] proves that all hierarchies for ternary voting games are achievable in the class of $I$-complete games. The purpose of this section is to prove that all hierarchies are achievable in the subclass of zero-centered strongly weighted games. For that, we will need the following result that was proved in [18], that establishes some links between weights and desirability relations for $(3,2)$ games.

Proposition 4.2 Given two arbitrary players $p$ and $r$ in a weighted $(3,2)$ game, for any weight function representing it, we have:

(i) $w^{+}(p) \geq w^{+}(r) \Rightarrow p \succsim_{D^{+}} r$.

(ii) $-w^{-}(p) \geq-w^{-}(r) \Rightarrow p \succsim_{D^{-}} r$.

(iii) $w^{+}(p)-w^{-}(p) \geq w^{+}(r)-w^{-}(r) \Rightarrow p \succsim_{D^{ \pm}} r$.

Theorem 4.3 All I-hierarchies are achievable in the class of zero-centered strongly weighted $(3,2)$ games. 
Proof : Following Friedman et al. [20]'s result for simple games, all $D$-hierarchies are achievable for weighted simple games except the two sequences : $(m, 1,1,1)$ and $(m, 1,1)$ for $m \geq 1$. Hence, there exist suitable weighted $(2,2)$ games certifying all achievable hierarchies for $\succsim_{D}$. We assume in what follows that the weighted representations chosen for these weighted games assign the same weights to equally-desirable voters for the $\succsim_{D}$ relation, i.e. if $i \succsim_{D} j$ and $j \succsim_{D} i$ (or $i \approx_{D} j$ ) if and only if $w_{i}=w_{j}$.

Let $\left(m_{1}, \ldots, m_{t}\right)$ be a given hierarchy with $\sum_{k=1}^{t} m_{k}=n$ and different of $(m, 1,1,1)$ and $(m, 1,1)$ for all $m \geq 1$. Then it exists a weighted simple game with weighted representation $\left[Q ; w_{1}, w_{2}, \ldots, w_{n}\right]$ such that $w_{i}>w_{j}$ if and only if $i \succ_{D} j$ and $w_{i}=w_{j}$ if and only if $i \approx_{D} j$, and with $\left(m_{1}, \ldots, m_{t}\right)$ as a hierarchy.

From each of these weighted representations we define a zero-sum strongly weighted $(3,2)$ voting game as follows : the quota is $Q$ and the weights for voters are $w(i)=\left(w_{i}, 0,-w_{i}\right)$ for all $i \in N$.

If $i \succ_{D} j$ (equivalently to $w_{i}>w_{j}$ according to the selection of weights done) we have $V\left(S_{1} \cup\{i\}, S_{2} \backslash\{i\}, S_{3}\right) \geq V\left(S_{1} \cup\{j\}, S_{2} \backslash\{j\}, S_{3}\right)$ for all 3-partition with $i, j \in S_{2}$ since $w^{+}(i)=w_{i}>w_{j}=w^{+}(j)$ which implies $w\left(S_{1} \cup\{i\}, S_{2} \backslash\{i\}, S_{3}\right)=$ $\left(w_{i}-w_{j}\right)+w\left(S_{1} \cup\{j\}, S_{2} \backslash\{j\}, S_{3}\right)>w\left(S_{1} \cup\{j\}, S_{2} \backslash\{j\}, S_{3}\right)$. Note, moreover, that each minimal winning bipartition for the simple game $(S, N \backslash S)$ induces the minimal winning 3-partition $(S, N \backslash S, \emptyset)$ for the $(3,2)$ game, and as $i \succ_{D} j$ it exists at least a bipartition $(S, N \backslash S)$ with $i, j \notin S$ such that $w(S \cup\{i\},(N \backslash S) \backslash\{i\}) \geq Q$ and $w(S \cup\{j\},(N \backslash S) \backslash\{j\})<Q$. Thus, the induced 3-partition $(S, N \backslash S, \emptyset)$ with $i, j \notin S$ verifies $w\left(S_{1} \cup\{i\},(N \backslash S) \backslash\{i\}\right) \geq Q$ and $w\left(S_{1} \cup\{j\},(N \backslash S) \backslash\{j\}\right)<Q$. Hence, $i \succ_{D} j$ for the simple game if and only if $i \succ_{D^{+}} j$ for the $(3,2)$ game.

Furthermore, as the $(3,2)$ game is zero-centered strongly weighted, we have $i \succsim_{D^{ \pm}} j$ and $i \succsim_{D^{-}} j$ whenever $i \succ_{D} j$ (or, equivalently, $i \succ_{D^{+}} j$ ), since $w_{i}-$ $\left(-w_{i}\right)>w_{j}-\left(-w_{j}\right)$ and $0-\left(-w_{i}\right)>0-\left(-w_{j}\right)$ respectively.

If $i \approx_{D} j$ (equivalently to $w_{i}=w_{j}$ according to the selection of weights done) we have $i \approx_{D} j$ for the simple game if and only if $i \approx_{D^{+}} j, i \approx_{D^{-}} j$ and $i \approx_{D^{ \pm}} j$ if and only if $i \approx_{I} j$ for the $(3,2)$ game. In other words, relation $\succsim_{D}$ for the simple game coincides with the influence relation $\succsim_{I}$ for the $(3,2)$ game.

Now it remains to prove that the two sequences $(m, 1,1,1)$ and $(m, 1,1)$ for $m \geq 1$ are also achievable in the class of zero-centered strongly weighted $(3,2)$ games.

- Proof for $(m, 1,1,1)$.

Consider the zero-centered strongly game with $n$ voters with quota $Q=1$ 
and weights :

$w(a)=(1,0,-1), \quad w(b)=(2,0,-2), \quad w(c)=(3,0,-3), \quad w\left(d_{i}\right)=(4,0,-4)$

where $i=1, \ldots, n-3$. By Proposition 4.2 it follows :

$$
d_{i} \succsim_{I} c \succsim_{I} b \succsim_{I} a
$$

It only remains to prove that these rankings are strict. However, one may easily check, by choosing convenient 3-partitions that

$$
d_{i} \not_{D^{+}} c, \quad c \not_{D^{+}} b, \quad b \not_{D^{-}} a
$$

Hence,

$$
d_{i} \succ_{I} c \succ_{I} b \succ_{I} a
$$

and the $I$-hierarchy $(m, 1,1,1)$ is achievable for this zero-centered strongly weighted $(3,2)$ game.

- Proof for $(m, 1,1)$.

Consider the zero-centered strongly game with $n$ voters with quota $Q=2$ and weights :

$$
w(a)=(1,0,-1), \quad w(b)=(2,0,-2), \quad w\left(c_{i}\right)=(3,0,-3)
$$

where $i=1, \ldots, n-2$. By Proposition 4.2 it follows :

$$
c_{i} \succsim_{I} b \succsim_{I} a
$$

It only remains to prove that these rankings are strict. One may easily check, by choosing convenient 3-partitions that

$$
c_{i} \not D^{+} b, \quad b \not_{D^{+}} a
$$

Hence,

$$
c_{i} \succ_{I} b \succ_{I} a
$$

and the $I$-hierarchy $(m, 1,1)$ is achievable for this zero-centered strongly weighted $(3,2)$ game. 


\section{$5 \quad H$-hierarchies}

In this section, we partially address the problem of existence of achievable hierarchies for $H$-complete $(3,2)$ games.

The following notations are useful.

Notation 5.1 Given a subset $A$ of $N$, a voter $b \in A$, a 3-partition $S=\left(S_{1}, S_{2}, S_{3}\right)$ of $N, p, r \in N$ and $x \notin N$, we denote by :

- $\pi_{p r}$ the transposition of voters $p$ and rof $N$.

- $T=S \backslash\{r\}$ the 3-partition of $N \backslash\{r\}$ such that : $\forall i \in\{1,2,3\}, T_{i}=$ $\left\{\begin{array}{lll}S_{i} & \text { if } & r \notin S_{i} \\ S_{i} \backslash\{r\} & \text { if } & r \in S_{i}\end{array}\right.$

- $\mathcal{T}_{x}(S, A)$ the 3 -partition $T$ of $N \cup\{x\}$ such that :

$\left\{x \in T_{t}\right.$ where $t=\min _{i \in\{1,2,3\}}\left\{i: A \cap S_{i} \neq \emptyset\right\}$,

and the level of approval of every player of $N$ in $T$ is the same as in $S$.

- $\mathcal{T}_{x}(b, S, A)$ the 3-partition $T$ of $N \cup\{x\}$ such that :

$\left\{\begin{array}{l}x \in T_{i} \text { if } b \in S_{i} \text { and } b \in T_{k} \text { where } k=\min _{i \in\{1,2,3\}}\left\{i,(A \backslash\{b\}) \cap S_{i} \neq \emptyset\right\}, \\ \text { and the level of approval of every player of } N \backslash\{b\} \text { in } T \text { is the same as in } S .\end{array}\right.$

The 2-player case : It is obvious that when there are only two voters, the hierarchy $\approx$ is achievable meanwhile the strict hierarchy $\succ$ is not.

The 3-player case : This case is solved in the following result.

Proposition 5.2 For $n=3$, the H-hierarchies (3) and $(2,1)$ are achievable meanwhile $H$-hierarchies $(1,1,1)$ and $(1,2)$ are not achievable.

Proof : First, it is obvious that the following $H$-complete $(3,2)$ games achieve the $H$-hierarchies $(3)$ and $(2,1)$ respectively. Let $N=\{a, b, c\}$ :

for the hierarchy $(3)$, take $W^{m}=\{(a b c, \emptyset, \emptyset)\}$ and for $(2,1)$, consider the set $W^{m}=\{(a, b, c),(b, a, c)\}$.

Now, assume that there is a 3-player $(3,2)$ game with $N=\{a, b, c\}$ such that $a \succ_{H} b \succ_{H} c$. Then there exist three 3-partitions $A, B, C$ of $N$ such that :

$a \in A_{1} \cap B_{1}, b \in A_{2} \cap C_{1}, c \in B_{2} \cap C_{2}, A, B, C \in W$ and $\pi_{a b}(A), \pi_{a c}(B), \pi_{b c}(C) \notin$ $W$.

First case $: c \in A_{3}$ 
First, let us assume that $b \in B_{3}$. If $a \in C_{3}$, then $A=(a, b, c), B=(a, c, b)$ and $C=(b, c, a)$ which implies $\pi_{a b}(A)=\pi_{a c}(C) . \pi_{a c}(C) \in W$ since $C \in W$ and $a \succ_{H} c$ meanwhile $\pi_{a b}(A) \notin W$; it is a contradiction. If $a \in C_{2}$, then $A=(a, b, c)$, $B=(a, c, b)$ and $C=(b, a c, \emptyset)$. We will show in this condition that $b \approx_{D^{-}} c$. It suffices to show that $c \succsim_{D^{-}} b$. Let $S$ be a 3 -partition such that $b, c \in S_{3}$ and assume that $\left(S_{1}, S_{2} \cup\{b\}, S_{3} \backslash\{b\}\right) \in W$. Since none of the 3-partitions $(\emptyset, a b, c)$ and $(\emptyset, b, a c)$ is winning, we must have $\left(S_{1}, S_{2} \cup\{b\}, S_{3} \backslash\{b\}\right)=(a, b, c)$ and therefore $\left(S_{1}, S_{2} \cup\{c\}, S_{3} \backslash\{c\}\right)=(a, c, b)=B \in W$. Thus, $c \succsim_{D^{-}} b$ and by hypothesis, $b \succ_{H} c$ implying that $b \succsim_{D^{-}} c$. Finally, $b \approx_{D^{-}} c$ which is a contradiction. If $a \in C_{1}$, it can easily be shown that $b \approx_{D^{-}} c$, which is once again a contradiction.

Second, let us assume that $b \in B_{2}$. If $a \in C_{3}$, then $\pi_{a b}(A)=\pi_{a c}(C) \in W$ since $C \in W$ and $a \succ_{H} c$. However, $\pi_{a b}(A) \notin W$ and this is a contradiction. If $a \in C_{2}$, then we have $a \approx_{D^{-}} c$, which is a contradiction. If $a \in C_{1}$, then we have $a \approx_{D^{-}} c$, which is also a contradiction.

Finally, let us assume that $b \in B_{1}$. If $a \in C_{3}$, then $\pi_{a b}(A)=\pi_{a c}(C) \in W$ since $C \in W$ and $a \succ_{H} c$. However, $\pi_{a b}(A) \notin W$ and this is a contradiction. If $a \in C_{2}$, then the contradiction follows and if $a \in C_{1}$, then $\pi_{b c}(C) \supset A \in W$. It is a contradiction.

Second case $: c \in A_{2} \cup A_{1}$.

If $a \in C_{3} \cup C_{2}$, then it can be checked that $\pi_{a b}(A) \supseteq C \in W$, which is a contradiction because $\pi_{a b}(A) \notin W$.

If $a \in C_{1}$, then $\pi_{b c}(C) \supseteq A \in W$, a contradiction because $\pi_{b c}(C) \notin W$.

Hence, for $n=3$ the $H$-strict hierarchy is not achievable.

We can prove analogously that the $H$-hierarchy $(1,2)$ is not achievable.

We give in Table 1 some examples of strongly weighted $(3,2)$ games and the $H$-hierarchies induced by these games, where $W^{m}$ denotes the set of minimal winning tripartitions.

In the sequel we consider the cases $n \geq 4$. We need the following important lemma.

Lemma 5.3 If an $H$-hierarchy $\left(m_{1}, \ldots, m_{i}, \ldots, m_{t}\right)$ is achievable then the $H$-hierarchy $\left(m_{1}, \ldots, m_{i}+1, \ldots, m_{t}\right)$ is achievable as well.

Proof : Let $(N, W)$ be a $(3,2)$ game that achieves the $H$-hierarchy $\left(m_{1}, \ldots, m_{i}, \ldots\right.$, $\left.m_{t}\right)$. Let $A_{i}$ the set of the $m_{i}$ equivalent players in the preceding $H$-hierarchy and let $N^{\prime}=N \cup\{x\}$.

$$
W^{\prime}=\left\{T \in 3^{N^{\prime}}: \exists(b, S) \in A_{i} \times W, T=\mathcal{T}_{x}\left(S, A_{i}\right) \text { or } T=\mathcal{T}_{x}\left(b, S, A_{i}\right)\right\} .
$$




\begin{tabular}{|c|c|c|c|c|c|c|}
\hline$W^{m}$ & $Q$ & $w(a)$ & $w(b)$ & $w(c)$ & $w(d)$ & $H$-hierarchy \\
\hline$(a, b, c)$ and $(b, a, c)$ & 1 & $(1,0,-1)$ & $(1,0,-1)$ & $(0,0,0)$ & $x$ & $a \approx b \succ c$ \\
\hline $\begin{array}{l}(a, c d, b),(a, b, c d), \\
(b, a c, d),(b, a d, c) \\
(c d, a, b),(a c, \emptyset, b d), \\
\text { and }(a d, \emptyset, b c)\end{array}$ & 1 & $(5,0,-5)$ & $(3,0,-3)$ & $(2,0,-2)$ & $(2,0,-2)$ & $a \succ b \succ c \approx d$ \\
\hline $\begin{array}{l}(b, a c, d),(b, a d, c) \\
(a, c, b d),(c, a b, d) \\
(a, b, c d) \text { and }(c, a d, b)\end{array}$ & 0 & $(3,0,-3)$ & $(2,0,-2)$ & $(2,0,-2)$ & $(0,0,-1)$ & $a \succ b \approx c \succ d$ \\
\hline $\begin{array}{l}(a, b, c d),(a, d, b c) \\
(a, c, b d),(c d, a, b) \\
(b d, a, c) \text { and }(b c, a, d)\end{array}$ & 1 & $(3,0,-3)$ & $(1,0,-1)$ & $(1,0,-1)$ & $(1,0,-1)$ & $a \succ b \approx c \approx d$ \\
\hline
\end{tabular}

Table 1: Examples of achievable $H$-hierarchies.

We will show that $\left(N^{\prime}, W^{\prime}\right)$ achieves the $H$-hierarchy $\left(m_{1}, \ldots, m_{i}+1, \ldots, m_{t}\right)$.

Let $B_{i}=A_{i} \cup\{x\}$ and $B_{j}=A_{j}$ for all $j \in\{1, \ldots, i-1, i+1, \ldots, t\}$.

Let us consider $p \in B_{k}, r \in B_{j}, k \leq j$.

It is obvious that if $k=j$ then $p$ and $r$ are equivalent. In the sequel we assume with no loss of generality that $k<j$.

First case $: j \neq i$ and $k \neq i$.

There exists a 3-partition $S \in W$ such that $p \in S_{1}, r \in S_{2}$ and $\pi_{p r}(S) \notin W$, since $p \succ_{D^{+}} r$ in $(N, W)$.

We have $T=\mathcal{T}_{x}\left(S, A_{i}\right) \in W^{\prime}$. If $\pi_{p r}(T) \in W^{\prime}$, then $\pi_{p r}(T \backslash\{x\}) \in W$, which is a contradiction since $\pi_{p r}(T \backslash\{x\})=\pi_{p r}(S)$. Thus, $\pi_{p r}(T) \notin W^{\prime}$ and hence, $p \succ_{D^{+}} r$ in $\left(N^{\prime}, W^{\prime}\right)$. Similarly, we have $p \succ_{D^{-}} r$ and $p \succ_{D^{ \pm}} r$ in $\left(N^{\prime}, W^{\prime}\right)$.

Second case $: j=i$ and $k \neq i$.

First, assume that $r \neq x$. There exists a 3-partition $S \in W$ such that : $p \in S_{1}, r \in S_{2}$ and $\pi_{p r}(S) \notin W$, since $p \succ_{D^{+}} r$ in $(N, W) . T=\mathcal{T}_{x}\left(S ; A_{i}\right) \in W^{\prime}$. If $\exists a \in B_{i} \backslash\{x\}, a \in S_{1}$, then $\pi_{p r}(T) \in W^{\prime}$ implies that $\pi_{p r}(T \backslash\{x\}) \in W$, which is a contradiction since $\pi_{p r}(T \backslash\{x\})=\pi_{p r}(S)$. Thus, $\pi_{p r}(T) \notin W^{\prime}$. If $B_{i} \cap S_{1}=\varnothing$, then $B_{i} \cap \pi_{p r}\left(T_{1}\right)=\{r\}$ and $\pi_{p r}(T) \notin W^{\prime}$, ( see the definition of $W^{\prime}$ ). Hence, $p \succ_{D^{+}} r$ in $\left(N^{\prime}, W^{\prime}\right)$. Similarly, we have $p \succ_{D^{-}} r$ and $p \succ_{D^{ \pm}} r$ in $\left(N^{\prime}, W^{\prime}\right)$.

Second, assume that $r=x$. Let $b \in B_{i}: b \neq x$. There exists a 3-partition $S \in W$ such that $p \in S_{1}, b \in S_{2}$ and $\pi_{p b}(S) \notin W$, since $p \succ_{D^{+}} b$ in $(N, W)$. Then, $T=\mathcal{T}_{x}\left(b, S, A_{i}\right) \in W^{\prime}$. If $\exists a \in B_{i} \backslash\{x\}, a \in S_{1}$, then $\pi_{p x}(T) \in W^{\prime}$ implies $\pi_{p x}(T \backslash\{x\}) \in W$ ( by the definition of $W^{\prime}$ ). This is a contradiction since $\pi_{p x}(T \backslash\{x\})=\pi_{p b}(S)$, thus $\pi_{p x}(T) \notin W^{\prime}$. 
If $B_{i} \cap S_{1}=\emptyset$, then, $B_{i} \cap \pi_{p x}\left(T_{1}\right)=\{x\}$ and $\pi_{p x}(T) \notin W^{\prime}$ (by the definition of $\left.W^{\prime}\right)$. Hence, $p \succ_{D^{+}} x$ in $\left(N^{\prime}, W^{\prime}\right)$.

Likewise, $p \succ_{D^{-}} x$ and $p \succ_{D^{ \pm}} x$ in $\left(N^{\prime}, W^{\prime}\right)$.

Third case : If $j \neq i$ and $k=i$.

First, assume that $p \neq x$. There exists a 3-partition $S \in W$ such that $p \in S_{1}$, $r \in S_{2}$ and $\pi_{p r}(S) \notin W$ since $p \succ_{D^{+}} r$ in $(N, W)$. We have $T=\mathcal{T}_{x}\left(S, A_{i}\right) \in W^{\prime}$. If $\exists a \in B_{i} \backslash\{x, p\}, a \in S_{1}$, then $\pi_{p r}(T) \in W^{\prime}$ implying that $\pi_{p r}(T \backslash\{x\}) \in W$. This is a contradiction since $\pi_{p r}(T \backslash\{x\})=\pi_{p r}(S)$ : thus $\pi_{p r}(T) \notin W^{\prime}$. If $B_{i} \cap S_{1}=\{p\}$, Then $\pi_{p r}(T) \notin W^{\prime}$ ( see the definition of $W^{\prime}$ ) hence, $p \succ_{D^{+}} r$ in $\left(N^{\prime}, W^{\prime}\right)$. Likewise, $p \succ_{D^{-}} r$ and $p \succ_{D^{ \pm}} r$ in $\left(N^{\prime}, W^{\prime}\right)$.

Now assume that $p=x$. Let $b \in B_{i}, b \neq x$. There exists a 3-partition $S \in W$ such that $: b \in S_{1} ; r \in S_{2}$ and $\pi_{b r}(S) \notin W$, since $b \succ_{D^{+}} r$ in $(N, W)$. We have $T=\mathcal{T}_{x}\left(S, A_{i}\right) \in W^{\prime}$. If $\exists a \in B_{i} \backslash\{x, b\}, a \in S_{1}$, then $\pi_{x r}(T) \in W^{\prime}$ implies $S^{\prime} \in W$, where $S^{\prime}$ is the 3-partition of $N$ such that $: b \in S_{2}^{\prime}, r \in S_{1}^{\prime}$ and every player of $N \backslash\{b, r\}$ has the same level of approval in $S^{\prime}$ and $\pi_{x r}(T)$. This is a contradiction since $S^{\prime}=\pi_{b r}(S)$; thus $\pi_{x r}(T) \notin W^{\prime}$.

If $B_{i} \cap S_{1}=\{b\}$, then $\pi_{x r}(T) \notin W^{\prime}$ ( see definition of $W^{\prime}$ ). Hence, $x \succ_{D^{+}} r$ in $\left(N^{\prime}, W^{\prime}\right)$.

Similarly, we have $x \succ_{D^{-}} r$ and $x \succ_{D^{ \pm}} r$ in $\left(N^{\prime}, W^{\prime}\right)$.

Proposition 5.4 For $n=4$, any $H$-hierarchy distinct from $(1,1,1,1)$ and $(2,1,1)$ is achievable.

Proof : - The $H$-hierarchies $(4),(3,1)$, and $(2,2)$ are achievable thanks to the lemma 5.3 above and the fact that $(3)$ and $(2,1)$ are achievable $H$-hierarchies.

- It can be seen from table 1 above that the $H$-hierarchies $(1,3),(1,2,1)$ and $(1,1,2)$ are achievable.

Proposition 5.5 For $n \geq 5$, the strict $H$-hierarchy $(1,1, \ldots, 1)$ is achievable.

Proof : Assume that $n \geq 5$ and consider the following $(3,2)$ game $(N, W)$ where $N=\left\{x_{1}, x_{2}, \ldots, x_{n-1}, x_{n}\right\}$ and the set of minimal winning 3 -partitions is given by :

$$
W^{m}=\left\{\begin{aligned}
& \left(\left\{x_{a}\right\}, N \backslash\left\{x_{a}, x_{b}\right\},\left\{x_{b}\right\}\right), a, b \in\{1, \ldots, n\} a<b, b \notin\{a+2 ; a+3: a \leq n-3\} ; \\
& \left(\left\{x_{i}\right\}, N \backslash\left\{x_{i}, x_{i+2}, x_{i+3}\right\},\left\{x_{i+2}, x_{i+3}\right\}\right), i=1, \ldots, n-3 ; \\
& \left(\left\{x_{n-2}, x_{n-4}\right\}, N \backslash\left\{x_{n-4}, x_{n-3}, x_{n-2}, x_{n}\right\},\left\{x_{n}, x_{n-3}\right\}\right) ; \\
& \left(\left\{x_{n-1}\right\}, N \backslash\left\{x_{n-1}\right\}, \emptyset\right) ; \\
& \left(\left\{x_{3}, x_{4}, x_{5}\right\}, N \backslash\left\{x_{2}, x_{3}, x_{4}, x_{5}\right\},\left\{x_{2}\right\}\right)
\end{aligned}\right\} .
$$

It is obvious that the game above achieves the strict $H$-hierarchy

$\left(x_{1} \succ x_{2} \succ \ldots \succ x_{n-1} \succ x_{n}\right)$. 
Theorem 5.6 For all $t \in \mathbb{N}, t \geq 5$, the H-hierarchy $\left(m_{1}, m_{2}, \ldots, m_{t}\right)$ with $m_{i} \geq 1,1 \leq i \leq t$ is achievable.

Proof : Considering the preceding Proposition and Lemma 5.3, the proof of this theorem is straighforward.

The study of $H$-hierarchies can be summarized as follows.

- The $H$-hierarchies $(1,1),(1,2)$ and $(1,1,1)$ are not achievable.

- Nothing have been said for $H$-hierarchies $(1,1,1,1)$ and $(m, 1,1)$ for any $m \geq 2$ and

- All other $H$-hierarchies are achievable.

\section{Conclusion}

The paper at hand deals with voting rules with abstention also called $(3,2)$ games, a voting game that strictly includes the class of simple games. The $I$-influence relation introduced by Tchantcho et al [31] is a generalization of the desirability relation originally defined in simple games. It coincides with the extension of Shapley-Shubik and Banzhaf and Coleman indices defined by Felsenthal and Machover [9] and later on reconsidered in a general framework by Freixas ([13] and [14]) in the class of swap-robust $(3,2)$ games. Unlike in simple games, all hierarchies are achievable in the class of $I$-complete $(3,2)$ games, that is, in the class of swap-robust games. Moreover, given any hierarchy, there exists a basic weighted $(3,2)$ game that achieves that hierarchy.

In this paper, we point out some achievable and non achievable hierachies in the class of $H$-complete $(3,2)$ games, a class of game recently introduced by Freixas et al [18] in order to handle some shortcoming of the $I$-influence, namely the fact that there exist weighted $(3,2)$ games that are not $I$-complete. When the number of voters $n$, is two or three, the strict hierarchy is never achieved. However, for $n \geq 5$, the strict hierarchy $(1,1, \ldots, 1)$ is achievable. Any hierarchy with at least four strict relations (that is, $\succ$ ) is achievable. We did not succeed in determining whether the $H$-hierarchies $(1,1,1,1)$ and $(m, 1,1)$ for any $m \geq 2$ are achievable or not. The $H$-hierarchies $(1,1),(1,2)$ and $(1,1,1)$ are not achievable meanwhile all other $H$-hierarchies are achievable.

Even though a complete characterization of achievable $I$-hierarchies has been determined, the general question of the characterization of achievable $H$-hierarchies is still open. Furthermore, it could be interesting looking for the smallest class of VGA in which $H$-hierarchies are achievable. This will lead to refinement of 
some results obtained in this paper for $H$-hierarchies. As mentioned in the introduction, for simple games, Bishnu and Roy [4] have shown how to use minimal winning coalitions to extract the hierarchy of players. The same techniques could be investigated for extracting $I$-hierarchies on one hand and $H$-hierarchies on the other hand for voting games with abstention.

Acknowledgements : The authors thank sincerely two anonymous referees and the editor for their helpful comments.

\section{References}

[1] J.M. Alonso-Meijide, J.M. Bilbao, B. Casas-Méndez and J.R. Fernández. Weighted multiple majority games with unions: Generating functions and applications to the European Union. European Journal of Operational Research 198: 530-544, 2009.

[2] J. Banzhaf. Weighted voting doesn't work : a mathematical analysis Rutgers Law Review 58 : 317-343, 1965.

[3] D. Bean and J. Friedman and C. Parker. Simple majority achievable hierarchies. Theory and Decision 65 : 285-302, 2008.

[4] M. Bishnu and S. Roy. Hierarchy of players in swap robust voting games. Social Choice and Welfare 38 : 11-22, 2012.

[5] F. Carreras and J. Freixas. Complete simple games. Mathematical Social Sciences 32 : 139-155, 1996.

[6] J. Coleman. Control of collectivities and the power of a collectivity to act. In B. Lieberman (Ed.), Social choice pp. 269-300. New York : Gordon and Breach, 1971.

[7] W.D. Cook. Distance-based and ad hoc consensus models in ordinal preference ranking. European Journal of Operational Research 172 : 369-385, 2006 .

[8] L. Diffo Lambo and J. Moulen. Ordinal equivalence of power notions in voting games. Theory and Decision 53 : 313-325, 2002.

[9] D.S. Felsenthal, M. Machover. Ternary voting games. International. Journal of Game Theory 26 : 335-351, 1997. 
[10] D.S. Felsenthal, M. Machover. The measurement of voting power : Theory and practice, problems and paradoxes. Edward Elgar Publishing Ltd., Cheltenham, UK and Northampton, MA, USA, 1998.

[11] P.C. Fishburn. The theory of social choice. Princeton University Press, Princeton, 1973.

[12] J. Freixas and W.S. Zwicker. Weighted voting, abstention, and multiple levels of approval. Social Choice and Welfare 21 : 399-431, 2003.

[13] J. Freixas. The Shapley-Shubik power index for games with several levels of approval in the input and output. Decision Support Systems 39 : 185-195, 2005.

[14] J. Freixas. The Banzhaf index for games with several levels of approval in the input and output. Annals of Operations Research 137 : 45-66, 2005.

[15] J. Freixas. Probabilistic power indices for voting rules with abstention. Mathematical Social Sciences 64 : 89-99, 2012.

[16] J. Freixas, D. Marciniak and M. Pons. On the ordinal equivalence of the Johnston, Banzhaf and Shapley power indices. European Journal of Operational Research 216 : 367-375, 2012.

[17] J. Freixas and M. Pons. Hierarchies achievable in simple games. Theory and Decision 68 : 393-404, 2010.

[18] J. Freixas, B. Tchantcho and N. Tedjeugang. Voting game with abstention : linking completeness and weightedness. Decision Support Systems, 2013 http://dx.doi.org/10.1016/j.dss.2013.08.0152012.

[19] J. Freixas and W. Zwicker. Anonymous yes-no voting with abstention and multiple levels of approval. Games and Economic Behavior 69 : 428-444, 2009 .

[20] J. Friedman, L. McGrath, and C. Parker. Achievable hierarchies in voting games. Theory and Decision 61 : 305-318, 2006.

[21] J.R. Isbell. A class of simple games. Duke Mathematical Journal 25 : 423-439, 1958.

[22] G. Levitin. Optimal allocation of multi-state elements in linear consecutively connected systems with vulnerable nodes. European Journal of Operational Research 150 : 406-419, 2003. 
[23] W. Obata and H. Ishii. A method for discriminating efficient candidates with ranked voting data. European Journal of Operational Research 151 : 233-237, 2003.

[24] C. Parker. The influence relation for ternary voting games. Games and Economic Behavior 75 : 867-881, 2012.

[25] R. Pongou, B. Tchantcho and L. Diffo Lambo. Political infuence in multi-choice institutions : cyclicity, anonymity, and transitivity. Theory and Decision 70 : 157-178, 2011.

[26] A. Rubinstein. Stability of decision systems under majority rule Journal of Economic Theory 23 : 150-159, 1980.

[27] L.S. Shapley and M. Shubik. A method for evaluating the distribution of power in a committee system. American Political Science Review 48 : 787-792, 1954.

[28] T. Sueyoshi, J. Shang and W-C. Chiang. A decision support framework for internal audit priorization in a rental car company: A combined use between DEA and AHP. European Journal of Operational Research 199 : 219-231, 2009 .

[29] A.D. Taylor. Mathematics and politics : Strategy, voting, power and proof. Springer, Berlin, Germany 1995.

[30] A.D. Taylor and W.S. Zwicker. Simple games : desirability relations, trading, and pseudoweightings. Princeton University Press, New Jersey, USA, 1999.

[31] B. Tchantcho, L. Diffo Lambo, R. Pongou, and B. Mbama Engoulou. Voters' power in voting games with abstention : Influence relation and ordinal equivalence of power theories. Games and Economic Behavior 64 : 335-350, 2008.

[32] W.S. Zwicker. Anonymous voting rules with abstention : weighted voting. In S.J. Brams, W.V. Gehrlein, and F.S. Roberts, editors, The Mathematics of Preference, Choice, and Order : Essays in Honor of Peter C. Fishburn, pages 239-258. Springer, Heidelberg, 2009. 